\title{
Small-scale Fading and Delay in Conference Room with 802.11 Coverage Problems
}

\author{
F. Heereman, W. Joseph, E. Tanghe, D. Plets and L. Martens \\ Department of Information Technology, Ghent University/IBBT \\ Gaston Crommenlaan 8 bus 201, B-9050 Ghent, Belgium \\ Email: frederic.heereman@intec.ugent.be
}

\begin{abstract}
Small-scale fading and propagation delay have been characterized for the $2.4 \mathrm{GHz}$ band in a specific conference room with 802.11 coverage problems and a more typical conference room for comparison. The measurements were executed with a virtual SIMO (Single-Input Multiple-Output) system. The implications on the 802.11 performance have been investigated in terms of received signal strength and intersymbol interference. A higher reverberation time was found in the room with coverage problems, which causes a higher chance of intersymbol interference.
\end{abstract}

\section{INTRODUCTION}

In literature, delay properties of indoor wireless channels are mainly investigated for typical environments like offices, factories and colleges [1]. This paper is focused on a specific conference room where repeated coverage problems were reported with an 802.11 conference system. This system has a SISO (Single-Input Single-Output) configuration. According to the manufacturer, these problems only occur in this conference room and cannot be attributed to interference sources after spectral analysis.

In this paper, small-scale fading and propagation delay are characterized for the $2.4 \mathrm{GHz}$ band in the conference room with coverage problems (room A) and in another more typical conference room (room B) for comparison. Implications on the performance of 802.11 systems are investigated in terms of reduced signal strength and intersymbol interference (ISI).

\section{MeAsurements}

Measurements were executed in room A and B with a virtual SIMO (Single-Input Multiple-Output) system. In this setup, the Tx and Rx antenna, both broadband omnidirectional Electro-Metrics antennas of type EM-6116, were connected to a Rohde \& Schwarz ZVR vector network analyzer, which measured the scattering parameter $S_{21}$ as a function of the frequency. A coaxial cable with two amplifiers was used to realize the required Tx-Rx separation. The position of the $\mathrm{Rx}$ antenna, attached to a frame of BiSlides, was controlled by a laptop.

The measurements were done in the frequency range 2.5 - $3 \mathrm{GHz}$, which is out of the $2.4 \mathrm{GHz}$ band to exclude interference sources. In room A, 801 frequency points were used, which allows to resolve power delay profiles for delays up to $1.6 \mu$ s (greater than the 802.11n Guard Interval of $800 \mathrm{~ns}$
[2]). This delay corresponds to a path length of about $475 \mathrm{~m}$. A $23 \times 23 \mathrm{Rx}$ array was used, with a separation of $1.5 \mathrm{~cm}$. This corresponds to 3.6 samples per $\lambda / 2$ (where $\lambda$ is the wavelength), and a total array dimension of $3 \lambda$. In room $B$, the following settings were used: 401 frequency points, a $4 \times 4$ $\mathrm{Rx}$ array with a separation of $4 \mathrm{~cm}$.

In room $\mathrm{A}$, the following positions of $\mathrm{Tx}$ and the $\mathrm{Rx}$ array were considered (Fig. 1): Tx at position 1 (usual position of the access point during meetings) and $\mathrm{Rx}$ at position 2 (chairman's seat on the conference table) (case 1a) (and vice versa (case $1 \mathrm{~b})$ ), and $\mathrm{Tx}$ at position 1 and $\mathrm{Rx}$ at position 3 (other seat at the conference table) (case 2a) (and vice versa (case 2b)). At position 1, the height of the antenna (Tx or $\mathrm{Rx}$ ) was always $1.8 \mathrm{~m}$, while at positions 2 and 3, the antenna height was always $1.2 \mathrm{~m}$. The distance between Tx and Rx for case $1 \mathrm{a}-\mathrm{b}$ is $8.9 \mathrm{~m}$, for case $2 \mathrm{a}-\mathrm{b} 6.9 \mathrm{~m}$.

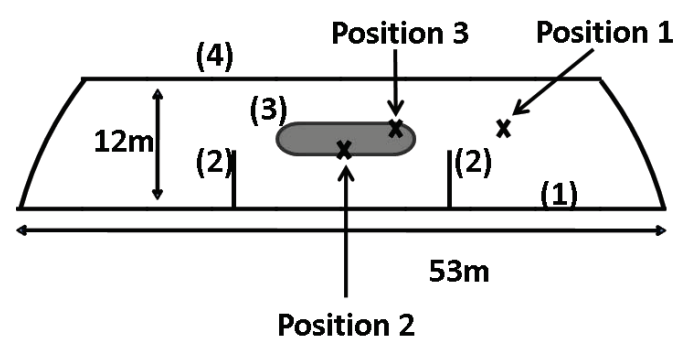

Fig. 1. Plan of conference room A. The conference table is indicated by (3). Measurements were executed at Tx/Rx positions $1-3$. The wall behind position 2 (indicated as (1)) and the two dividing walls (indicated as (2)) contain about 30 metal HVAC plates. The wall at the other side (indicated as (4)) consists of windows only.

The wall of room A behind position 2 (indicated as (1) in Fig. 1) and the two dividing walls (indicated as (2) in Fig. 1) contain about 30 metal HVAC (Heating, Ventilation, and Air Conditioning) plates (dimension $1 \mathrm{~m}$ by $1.5 \mathrm{~m}$ ). The wall at the other side (indicated as (4) in Fig. 1) consists of windows only. The ceiling, which looks like a part of an ellipsoid, contains a metal wire mesh, with a minimal separation of about $1 \mathrm{~cm}$. The dimensions of the room are $12 \mathrm{~m} \times 53 \mathrm{~m}$ and the ceiling has a maximal height of $13 \mathrm{~m}$.

Conference room B is cylinder-shaped with about $30 \mathrm{~m}$ diameter and a height of about $7 \mathrm{~m}$. Tx was positioned $1 \mathrm{~m}$ 
from the wall at a height of $2 \mathrm{~m}$ and the $\mathrm{Rx}$ array was set at two different positions (case 3a-b) near the middle of the room, at a height of $1.2 \mathrm{~m}$. The Tx-Rx separation is $18.4 \mathrm{~m}$ for case $3 \mathrm{a}$ and $15.3 \mathrm{~m}$ for case $3 \mathrm{~b}$. For all measurements (rooms $\mathrm{A}$ and $\mathrm{B})$, there was a line-of-sight condition.

\section{SMALL-SCALE FADING}

The performance of the wireless system can be reduced by small-scale fading. The ratio between $\mathrm{P}_{\mathrm{R}}[\mathrm{dBm}]$ (the power leaving the $\mathrm{Rx}$ terminals) and $\mathrm{P}_{\mathrm{T}}[\mathrm{dBm}]$ (the power entering the $\mathrm{Tx}$ terminals) is given by the measured scattering parameter $\mathrm{S}_{21}: P_{R}-P_{T}=20 \log \left(\left|S_{21}\right|\right)$, where $|\cdot|$ is the absolute value.

An estimation of the minimum required $\left|S_{21}\right|^{2}$ value can be made as follows. Taking into account the gains of the measurement $\mathrm{Rx}$ and $\mathrm{Tx}$ antenna $(1 \mathrm{dBi})$, the $\mathrm{Rx}$ gain of a realistic wireless system $(4 \mathrm{dBi})$, and a maximum allowed EIRP (Equivalent Isotropically Radiated Power) of $20 \mathrm{dBm}$, we estimate the received power of the wireless system as $\left|S_{21}\right|^{2}+22 \mathrm{dBm}$. The sensitivity of the $802.11 \mathrm{n}$ reference receiver is $-64 \mathrm{dBm}$ for $65 \mathrm{Mbps}$ (Packet Error Rate $<10 \%$ ) [2]. Hence, we estimate that there is good coverage when $\left|S_{21}\right|^{2}>-86 \mathrm{~dB}$.

The cumulative distribution function (CDF) of the measured $\left|S_{21}\right|^{2}$ samples is determined for the different cases (Fig. 2). Each CDF is based on all Rx positions (of the array) and all frequency points. The probability that $\left|S_{21}\right|^{2}<-86 \mathrm{~dB}$ is of the order of $10^{-5}-10^{-4}$ for cases $1 \mathrm{a}-\mathrm{b}$ and $2 \mathrm{a}-\mathrm{b}$, and of the order of $10^{-4}-10^{-3}$ for case $3 a-b$. These are low probabilities, but the position of deep fades (drops of $\left|S_{21}\right|^{2}$ ) can be easily influenced by little changes in the environment (e.g. people).

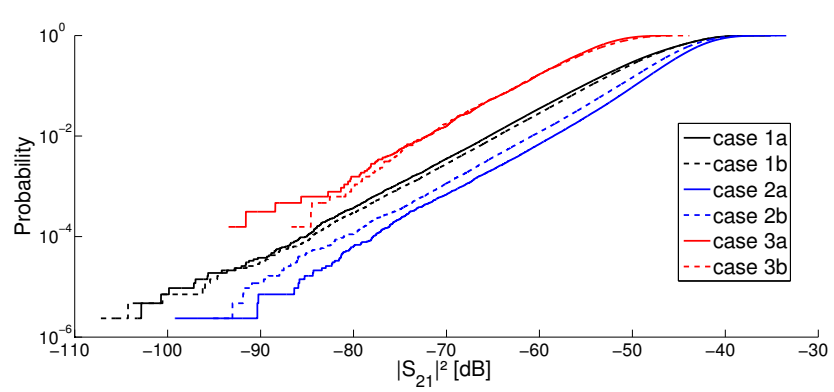

Fig. 2. CDF of $\left|S_{21}\right|^{2}$ for cases $1 \mathrm{a}-\mathrm{b}$ and 2a-b (room A) and case 3a-b (room B).

To compare the degree of small-scale fading in the CDFs of $\left|S_{21}\right|^{2}$ for the different cases, we should take into account that for higher Tx-Rx separation, the $\left|S_{21}\right|^{2}$ values are lower. Therefore, we plot the CDF of $\left|S_{21}\right|^{2}-\left(\left|S_{21}\right|^{2}\right)_{a v}$ (see Fig. 3), where $\left(\left|S_{21}\right|^{2}\right)_{a v}$ is the average of the (linearscaled) $\left|S_{21}\right|^{2}$ samples. Note that for low probability, the CDFs in Fig. 2 and 3 are less accurate due to the finite number of samples. The degree of small-scale fading is higher for case 1a than for case $2 \mathrm{a}$ (horizontal shift of the CDF tail of $8 \mathrm{~dB}$ at a probability of $10^{-3}$, see Fig. 3) and is also higher for case $1 \mathrm{~b}$ than for case $2 b$ (horizontal shift of the CDF tail of $4 \mathrm{~dB}$ at a probability of $10^{-3}$ ). The degree of small-scale fading of cases $3 a-b$ seems to lie between case $1 \mathrm{a}$ and case $2 \mathrm{a}$. The difference in degree of small-scale fading between case 1a-b (position 2 involved) and case $2 \mathrm{a}-\mathrm{b}$ (position 3 involved) can be explained by the fact that position 2 is closer to and more surrounded by metal HVAC plates than position 3. Note that in room A more coverage problems were reported at position 2 than at other seats at the conference table (e.g. position 3). This is in agreement with the higher degree of small-scale fading for cases $1 \mathrm{a}-\mathrm{b}$ (position 2 involved) than for cases $2 \mathrm{a}-\mathrm{b}$ (position 3 involved).

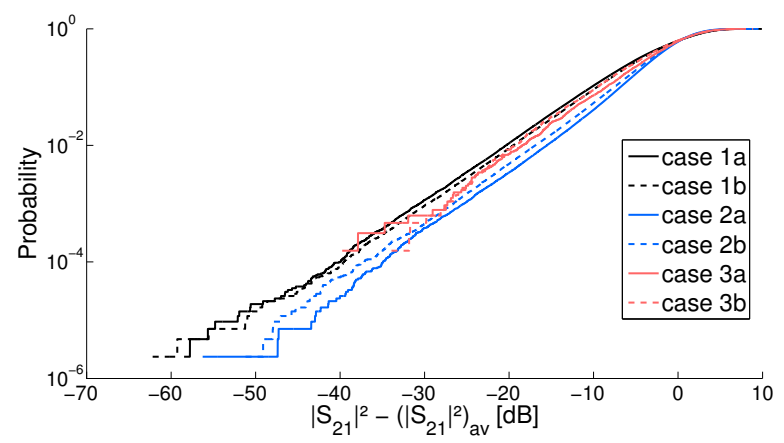

Fig. 3. CDF of $\left|S_{21}\right|^{2}-\left(\left|S_{21}\right|^{2}\right)$ av for cases 1a-b and 2a-b (room A) and case 3a-b (room B).

\section{DELAY}

In this section, the propagation delay is investigated with the focus on intersymbol interference (ISI). We assume a Guard Interval (GI) of $800 \mathrm{~ns}$ [3]. Based on the SIMO measurements, Averaged Power Delay Profiles (APDP) were made for all cases [4] (see Fig. 4). The resolution is $2 \mathrm{~ns}$ for all APDPs. The diffuse part of the APDP $[\mathrm{dB}]$ can be expressed as

$$
A P D P=C_{0}-10 \log (e)\left(\tau-\tau_{0}\right) / \tau_{r}
$$

where $\tau_{r}$ is the reverberation time (i.e. time over which the APDP is reduced by the exponential factor $e$ ) and $\tau_{0}$ is the delay of the first arriving path [5]. Thus, by definition, $\mathrm{C}_{0}$ is the power of the extrapolated diffuse part of the APDP, evaluated at $\tau_{0}$. Since for all cases, there is a line-of-sight condition, $\tau_{0}$ is $d / c$, where $d$ is the distance between $T x$ and $R x$ and $c$ the speed of light. The parameters $\tau_{r}$ and $\mathrm{C}_{0}$ are determined from the APDPs (Table I). We see that $\tau_{r}$ is a factor 2 higher in room A $(134-138 \mathrm{~ns})$ than in room $\mathrm{B}(55-59 \mathrm{~ns})$, while $\mathrm{C}_{0}$ is $5 \mathrm{~dB}$ higher in room $\mathrm{B}$ than in room $\mathrm{A}$. The higher $\tau_{r}$ in room A can be explained by the vicinity of many metal structures (HVAC plates, wire mesh in the ceiling).

The signal transfer (voltage over Tx terminals to voltage over Rx terminals) has a power reduction of $\left|S_{21}\right|^{2}$, measured at the carrier frequency (assuming that $\left|S_{21}\right|^{2}$ does not vary much over the bandwidth of the signal). Paths which arrive 


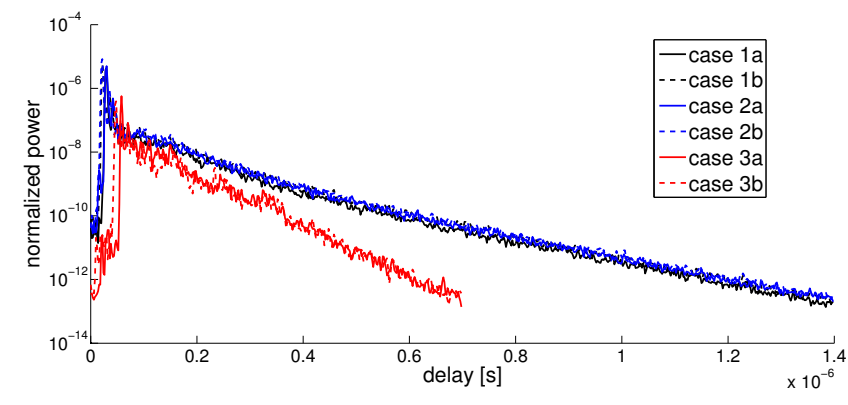

Fig. 4. Averaged power delay profiles for cases $1 \mathrm{a}-\mathrm{b}$ and $2 \mathrm{a}-\mathrm{b}$ (room A) and case 3a-b (room B).

TABLE I

PARAMETERS OF THE DIFFUSE PART OF THE APDPS FOR THE DIFFERENT CASES: REVERBERATION TIME $\tau_{r}$ AND $\mathrm{C}_{0}$. POWER REDUCTION $\mathrm{P}_{\mathrm{GI}}$ (DUE TO PATHS ARRIVING AT RX LATER THAN GI $+\tau_{0}$ ) (THEORETICAL ESTIMATION) IS COMPARED WITH $\left(\left|S_{21}\right|^{2}\right) a v$.

\begin{tabular}{|c|c|c|c|c|c|}
\cline { 2 - 6 } \multicolumn{1}{c|}{} & $\begin{array}{c}\tau_{r} \\
{[\mathrm{~ns}]}\end{array}$ & $\begin{array}{c}\mathrm{C}_{0} \\
{[\mathrm{~dB}]}\end{array}$ & $\begin{array}{c}\mathrm{P}_{\mathrm{GI}} \\
{[\mathrm{dB}]}\end{array}$ & $\begin{array}{c}\left(\left|S_{21}\right|^{2}\right)_{a v} \\
{[\mathrm{~dB}]}\end{array}$ & $\begin{array}{c}\mathrm{P}_{\mathrm{GI}}-\left(\left|S_{21}\right|^{2}\right)_{a v} \\
{[\mathrm{~dB}]}\end{array}$ \\
\hline case 1a & 135 & -83.0 & -56.7 & -45.0 & -11.7 \\
\hline case 1b & 134 & -82.2 & -56.0 & -43.9 & -12.1 \\
\hline case 2a & 138 & -82.6 & -55.4 & -45.1 & -10.3 \\
\hline case 2b & 137 & -81.5 & -54.8 & -43.0 & -11.8 \\
\hline case 3a & 58.5 & -77.0 & -91.6 & -53.7 & -37.9 \\
\hline case 3b & 54.9 & -77.0 & -96.0 & -52.8 & -43.2 \\
\hline
\end{tabular}

at $\mathrm{Rx}$ with a delay higher than $\tau_{0}+$ GI can cause ISI. The contribution of these paths can be worst-case estimated theoretically by a power reduction $\mathrm{P}_{\mathrm{GI}}$ of:

$$
P_{G I}=4 \exp \left(-G I / \tau_{r}\right) \frac{C_{0} M}{\tau_{r e s}^{2}} \tau_{r}^{2},
$$

where $\tau_{\text {res }}[\mathrm{s}]$ is the resolution of the APDP, and $\mathrm{C}_{0}$ and $\mathrm{P}_{\mathrm{GI}}$ are in linear scale. $\mathrm{M}$ is a margin due to constructive interference between paths with different angle of arrival. $M$ has been determined experimentally as follows. Based on the power delay profiles of all $\mathrm{Rx}$ positions, the maximum power is obtained as a function of the delay (Fig. 5). $\mathrm{M}$ is the difference between this maximum power and the averaged power delay profile. In this way, we find that the margin $\mathrm{M}$ is $9.5 \pm 0.5 \mathrm{~dB}$ for all cases. The power reduction $\mathrm{P}_{\mathrm{GI}}$ should be much lower than $\left|S_{21}\right|^{2}$ to exclude ISI [3]. Based on the experimentally determined parameters $\tau_{r}, \mathrm{C}_{0}$ and $\mathrm{M}$, we calculate $\mathrm{P}_{\mathrm{GI}}$ with (2). Here, a resolution $\tau_{\text {res }}$ of $3.3 \mathrm{~ns}$ is considered (see further). These $\mathrm{P}_{\mathrm{GI}}$ values should be compared with $\left(\left|S_{21}\right|^{2}\right)_{a v}: P_{G I}-\left(\left|S_{21}\right|^{2}\right)_{a v}=-12.1$ to $-10.3 \mathrm{~dB}$ for room $\mathrm{A}$ and -43.2 to $-37.9 \mathrm{~dB}$ for room $\mathrm{B}$. This is caused by the higher $\tau_{r}$ (factor 2 higher) for room A. Note that smallscale fading has been cancelled out in $\left(\left|S_{21}\right|^{2}\right)_{a v}$. If we take into account small-scale fading, fades higher than about $10 \mathrm{~dB}$ and $40 \mathrm{~dB}$ (relative to $\left(\left|S_{21}\right|^{2}\right)_{a v}$ ) can theoretically cause ISI for rooms A and B, respectively. This indicates that there is a greater chance of intersymbol interference in room $A$ than in room $\mathrm{B}$.

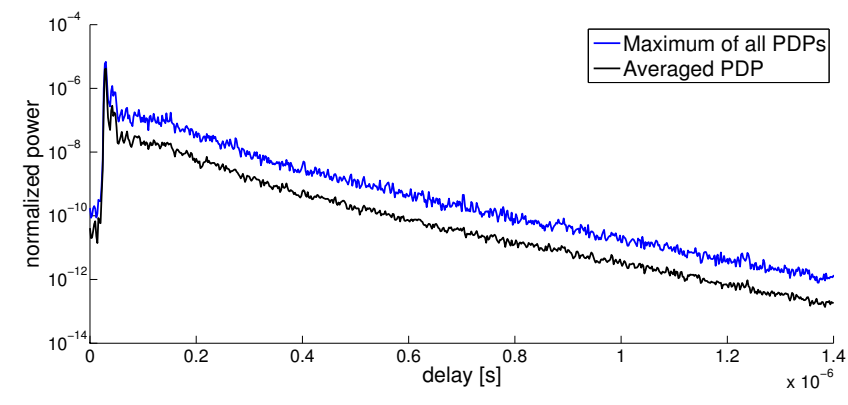

Fig. 5. Maximum of all power delay profiles (of all $\mathrm{Rx}$ positions) and averaged power delay profile (APDP) for case $1 \mathrm{~b}$ (room A). The vertical shift corresponds to the margin $\mathrm{M}: \mathrm{M}=9.5 \pm 0.5 \mathrm{~dB}$.

Equation (2) is a theoretical worst-case estimation of $\mathrm{P}_{\mathrm{GI}}$. However, it is possible to determine $\mathrm{P}_{\mathrm{GI}}$ as a function of frequency f per Rx array position fully experimentally. This is done by reconstructing the transfer function (voltage over Tx terminals to voltage over Rx terminals) corresponding to the paths which arrive at Rx with a delay lower than $\tau_{0}+$ GI. This reconstruction is done from the part of the impulse reponse (of which the absolute value gives the Power Delay Profiles (PDP)) with a delay $<\tau_{0}+$ GI. Thus, this transfer function is $S_{21}(f)-S_{21, \mathrm{GI}}(f)$ for paths arriving with a delay $<\tau_{0}+\mathrm{GI}$ at $\mathrm{Rx}$, where $S_{21, \mathrm{GI}}(f)$ is the transfer function corresponding to the paths which arrive with a delay $>\tau_{0}+\mathrm{GI}$ at $\mathrm{Rx}$. $\mathrm{P}_{\mathrm{GI}}$ is then $\left|S_{21, \mathrm{GI}}(f)\right|^{2}$. $\left|S_{21}(f)-S_{21, \mathrm{GI}}(f)\right|^{2}$ should be much higher than $\left|S_{21, \mathrm{GI}}(f)\right|^{2}$ to exclude ISI.

The reconstruction of the transfer function $S_{21}(f)-$ $S_{21, \text { GI }}(f)$ has been done for the frequency range $2.65 \mathrm{GHz}$ $-2.85 \mathrm{GHz}$. To minimize the influence of the window, used for the Fourier transformation in the calculation of the impulse response, we calculate for each measured frequency point an impulse response to reconstruct the transfer function around that frequency point. These PDPs are based on a lower frequency span (i.e. $300 \mathrm{MHz}$ ). Consequently, they have a bit higher resolution (i.e. $3.3 \mathrm{~ns}$ ), but have the same maximum delay, over which the PDP can be resolved. This maximum delay has to be higher than GI $(0.8 \mu \mathrm{s})$. This maximum delay is $1.6 \mu$ s for cases $1 \mathrm{a}-\mathrm{b}$ and $2 \mathrm{a}-\mathrm{b}$, and $0.8 \mu$ s for cases $3 \mathrm{a}-\mathrm{b}$. Therefore, we can do the reconstruction only for cases $1 a-b$ and $2 \mathrm{a}-\mathrm{b}$.

We investigate for cases $1 \mathrm{a}$ and $2 \mathrm{a}$ whether $\mid S_{21}(f)-$ $\left.S_{21, \mathrm{GI}}(f)\right|^{2}$ can be lower than $\left|S_{21, \mathrm{GI}}(f)\right|^{2}$, for the $23 \times 23$ $\mathrm{Rx}$ positions and all frequency points in the range $2.65 \mathrm{GHz}$ $2.85 \mathrm{GHz}$ (namely 321 frequency points). In this case, symbol errors due to ISI can be expected. For case 1a, this occurs 7 times (out of 169,809 samples). Here, the minimal (worst) ratio $\left|S_{21}(f)-S_{21, \mathrm{GI}}(f)\right|^{2}-\left|S_{21, \mathrm{GI}}(f)\right|^{2}$ is $-8.5 \mathrm{~dB}$. This situation is illustrated in Fig. 6, where $\left|S_{21}(f)-S_{21, \mathrm{GI}}(f)\right|^{2}$ and $\left|S_{21, \mathrm{GI}}(f)\right|^{2}$ are shown (in a small frequency range of $10 \mathrm{MHz}$ ). For case $2 \mathrm{a}$, this occurs 8 times. Here, the minimal (worst) ratio $\left|S_{21}(f)-S_{21, \mathrm{GI}}(f)\right|^{2}-\left|S_{21, \mathrm{GI}}(f)\right|^{2}$ is even $21.0 \mathrm{~dB}$ (Fig. 7). This shows that when (small-scale) fades 
are deep enough, the contribution of the paths arriving with a delay $>\tau_{0}+$ GI can exceed the contribution of the paths arriving with a delay $<\tau_{0}+$ GI, which results in ISI. From Figs. 6 and 7, we see that fades of $30-40 \mathrm{~dB}$ (compared to $\left(\left|S_{21}\right|^{2}\right)_{a v}$ level) can cause intersymbol interference. This is in agreement with the theoretical estimation in Table I (most right column, case $1 \mathrm{a}$ and $2 \mathrm{a}$ ), which says that intersymbol interference can occur for fades deeper than about $10 \mathrm{~dB}$.

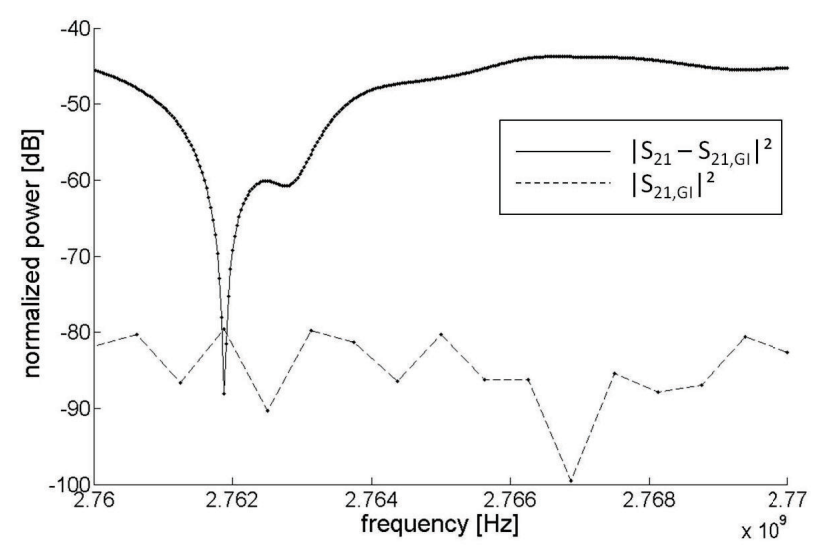

Fig. 6. Power reduction $\left|S_{21}(f)-S_{21, \mathrm{GI}}(f)\right|^{2}$, corresponding to the paths which arrive at $\mathrm{Rx}$ with a delay $<\tau_{0}+\mathrm{GI}$ : solid line. Power reduction $\left|S_{21, \mathrm{GI}}(f)\right|^{2}$, corresponding to the paths which arrive at Rx with a delay $>$ $\mathrm{GI}+\tau_{0}$ : dashed line. This is for case 1a (room A). The markers indicate the calculated points.

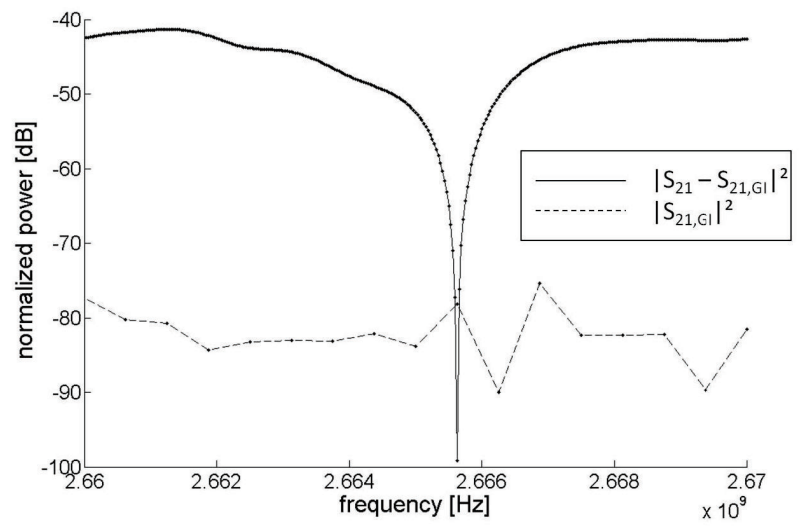

Fig. 7. Power reduction $\left|S_{21}(f)-S_{21, \mathrm{GI}}(f)\right|^{2}$, corresponding to the paths which arrive at $\mathrm{Rx}$ with a delay $<\tau_{0}+\mathrm{GI}$ : solid line. Power reduction $\left|S_{21, \mathrm{GI}}(f)\right|^{2}$, corresponding to the paths which arrive at Rx with a delay $>$ $\mathrm{GI}+\tau_{0}$ : dashed line. This is for case $2 \mathrm{a}$ (room A). The markers indicate the calculated points.

\section{Conclusions}

Small-scale fading and propagation delay have been characterized for the $2.4 \mathrm{GHz}$ band in a specific conference room (room A) with 802.11 coverage problems and a more typical conference room (room B) for comparison. The measurements were executed with a virtual SIMO system. The implications on the 802.11 performance have been investigated in terms of received signal strength and intersymbol interference.

At the chairman's seat in room A (where most coverage problems were reported), an $8 \mathrm{~dB}$ higher degree of small-scale fading was found compared to another seat on the conference table. Power delay profiles revealed that the reverberation time $\tau_{r}$ in room $\mathrm{A}$ is a factor 2 higher than in room $\mathrm{B}$. These findings could be explained by the vicinity of many metal structures in room A, especially near the chairman's seat.

We showed that the propagation properties of room A can result in coverage problems due to a reduced received signal strength as well as intersymbol interference. Due to the higher $\tau_{r}$ in room A, intersymbol interference can theoretically occur for (small-scale) fades higher than $10 \mathrm{~dB}$ and $40 \mathrm{~dB}$ in rooms $\mathrm{A}$ and $\mathrm{B}$, respectively.

\section{ACKNOWLEDGMENT}

This work was supported by the OMUS (Optimizing Multimedia Service Delivery) project, co-funded by the IBBT (Interdisciplinary institute for BroadBand Technology), a research institute founded by the Flemish Government in 2004, and the involved companies and institutions. W. Joseph is a postdoctoral fellow of the FWO-Flanders.

\section{REFERENCES}

[1] M. Awad, K. Wong, and Z. bin Li, "An integrated overview of the open literature's empirical data on the indoor radiowave channel's delay properties," IEEE Transactions on Antennas and Propagation, vol. 56, no. 5, pp. 1451-1468, 2008.

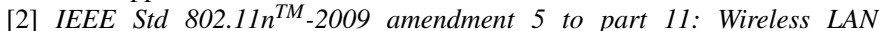
Medium Access Control (MAC) and Physical Layer (PHY) specifications: enhancements for higher throughput, IEEE Std., Oct 2009.

[3] Wikipedia. [Online]. Available: en.wikipedia.org/wiki/Guard \_interval

[4] E. Tanghe, W. Joseph, J. De Bruyne, L. Verloock, and L. Martens, "The industrial indoor channel: statistical analysis of the power delay profile," International Journal of Electronics and Communications, vol. 64, no. 9, pp. 806-812, 2010.

[5] J. Andersen, J. Nielsen, G. Pedersen, G. Bauch, and M. Herdin, "Room electromagnetics," IEEE Antennas and Propagation Magazine, vol. 49, no. 2, pp. 27-33, 2007. 